Lecture Notes in Computer Science

Edited by G. Goos, J. Hartmanis, and J. van Leeuwen 
Christel Baier Boudewijn R. Haverkort Holger Hermanns Joost-Pieter Katoen Markus Siegle (Eds.)

\title{
Validation of Stochastic Systems
}

\author{
A Guide to Current Research
}




\section{Series Editors}

Gerhard Goos, Karlsruhe University, Germany

Juris Hartmanis, Cornell University, NY, USA

Jan van Leeuwen, Utrecht University, The Netherlands

\section{Volume Editors}

Christel Baier

University of Bonn, Institute of Informatics I

Römerstr. 164, 53117 Bonn, Germany

E-mail: baier@cs.uni-bonn.de

\section{Boudewijn R. Haverkort}

University of Twente, Dept. of Computer Science and Electrical Engineering,

Design and Analysis of Communication Systems

P.O. Box 217, The Netherlands

E-mail: brh@cs.utwente.nl

\section{Holger Hermanns}

Saarland University, Dept. of Computer Science, Dependable Systems and Software 66123 Saarbrücken, Germany

E-mail: hermanns@cs.uni-sb.de

Joost-Pieter Katoen

University of Twente, Dept. of Computer Science, Formal Methods and Tools P.O. Box 217, 7500 AE Enschede, The Netherlands

E-mail: katoen@cs.utwente.nl

\section{Markus Siegle}

University of Federal Armed Forces Munich, Dept. of Computer Science 85577 Neubiberg, Germany

E-mail: siegle@informatik.unibw-muenchen.de

Library of Congress Control Number: 2004110613

CR Subject Classification (1998): F.1, F.3, D.2, D.4, I.6, C.1

ISSN 0302-9743

ISBN 3-540-22265-0 Springer Berlin Heidelberg New York

This work is subject to copyright. All rights are reserved, whether the whole or part of the material is concerned, specifically the rights of translation, reprinting, re-use of illustrations, recitation, broadcasting, reproduction on microfilms or in any other way, and storage in data banks. Duplication of this publication or parts thereof is permitted only under the provisions of the German Copyright Law of September 9, 1965, in its current version, and permission for use must always be obtained from Springer. Violations are liable to prosecution under the German Copyright Law.

Springer is a part of Springer Science+Business Media

springeronline.com

(C) Springer-Verlag Berlin Heidelberg 2004

Printed in Germany

Typesetting: Camera-ready by author, data conversion by Scientific Publishing Services, Chennai, India Printed on acid-free paper SPIN: 11015703 $06 / 3142$

543210 


\section{Preface}

It is with great pleasure that we present to you this tutorial volume entitled Validation of Stochastic Systems. It is one of the results of the Dutch-German bilateral cooperation project "Validation of Stochastic Systems" (VOSS), financed by NWO and DFG (the Dutch and German science foundations, respectively).

In the early days of 2002, the idea emerged to organize a seminar at Schloss Dagstuhl, not the usual Dagstuhl seminar with primarily invited participants, but a seminar aimed at young(er) people, and for which the organizers assign themes to be worked upon and presented on. Following an open call announced via the Internet in the spring of 2002, we received many applications for participation. After a selection procedure, we decided to assign (mostly) teams of two researchers to work on specific topics, roughly divided into the following four theme areas: "Modelling of Stochastic Systems," "Model Checking of Stochastic Systems," "Representing Large State Spaces," and "Deductive Verification of Stochastic Systems." These are the titles of the four parts of this volume.

The seminar was held in Schloss Dagstuhl during December 8-11, 2002 as part of the so-called GI/Research Seminar series. This series of seminars is financially supported by the Gesellschaft für Informatik, the German Computer Society. At that point in time the papers had already undergone a first review round. Each of the tutorial papers was presented in a one-hour session, and on the basis of the presentations we decided to bring together a selection of them into a book. A second review round was performed throughout 2003; at the end of 2003 all contributions were finished. We are glad that Springer-Verlag was willing to publish it in their well-established Lecture Notes in Computer Science series, in particular in the "green cover" Tutorial subseries.

To conclude this preface, we would like to thank NWO and DFG for making the VOSS bilateral cooperation project possible in the first place. Secondly, we would like to thank the Gesellschaft für Informatik for supporting the participants of the seminar. We would like to thank the whole team at Schloss Dagstuhl for their willingness to host us and for their hospitality. We also thank the authors of the tutorial papers as well as the reviewers for their efforts; without you, there would not have been a workshop! Finally, we would like to thank José Martínez (of the University of Twente) for his work on the editing of this volume.

Christel Baier Boudewijn Haverkort Holger Hermanns Joost-Pieter Katoen Markus Siegle 


\section{Table of Contents}

\section{Modelling Stochastic Systems}

Probabilistic Automata: System Types, Parallel Composition and

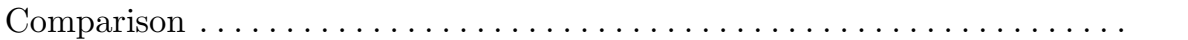

Ana Sokolova, Erik P. de Vink

Tutte le Algebre Insieme: Concepts, Discussions and Relations of Stochastic Process Algebras with General Distributions . . . . . . . . . . . . . 44 Mario Bravetti, Pedro R. D'Argenio

An Overview of Probabilistic Process Algebras and their Equivalences ... Natalia López, Manuel Núñez

\section{Model Checking of Stochastic Systems}

Verifying Qualitative Properties of Probabilistic Programs . . . . . . . . . 124 Benedikt Bollig, Martin Leucker

On Probabilistic Computation Tree Logic . . . . . . . . . . . . . . . . . . . . . . 147 Frank Ciesinski, Marcus Größer

Model Checking for Probabilistic Timed Systems . . . . . . . . . . . . . . 189 Jeremy Sproston

\section{Representing Large State Spaces}

Serial Disk-based Analysis of Large Stochastic Models . . . . . . . . . . . . 230 Rashid Mehmood

Kronecker Based Matrix Representations for Large Markov Models . . . . . 256 Peter Buchholz, Peter Kemper

Symbolic Representations and Analysis of Large Probabilistic Systems . . 296 Andrew Miner, David Parker

Probabilistic Methods in State Space Analysis . . . . . . . . . . . . . . . 339 Matthias Kuntz, Kai Lampka

\section{Deductive Verification of Stochastic Systems}

Analysing Randomized Distributed Algorithms . . . . . . . . . . . . . . 384 Gethin Norman 
An Abstraction Framework for Mixed Non-deterministic and Probabilistic Systems .

Michael Huth

The Verification of Probabilistic Lossy Channel Systems

Philippe Schnoebelen

Author Index 